\title{
The Influence of Nerve Fiber Distribution on the Recruitment Behavior resulting from Intraneural Stimulation
}

\author{
Jos P.A. Smit, Wim L.C. Rutten, Member, IEEE, Theo A. Frieswijk, and Ronald Busschers \\ Institute of Biomedical Technology, Faculty of Electrical Engineering, University of Twente \\ P.O. Box 217, 7500 AE Enschede, The Netherlands
}

\begin{abstract}
Force recruitment curves have been recorded for the rat extensor digitorum longus muscle (EDL) using monopolar stimulation with intraneural wire electrodes. The recruitment curves vary with the position of the electrode in the nerve. This has implications when intraneural stimulation techniques are used to precisely control muscle force. To assess this effect, two parameters were extracted for each recruitment curve: the threshold current and the slope in the low-force range. When these experimental parameters are compared with the same parameters resulting from simulated recruitment curves, it becomes clear that the low density of nodes of Ranvier and the degree of clustering of nerve fibers are of major importance for the recruitment behavior resulting from intraneural stimulation. It is also shown that the external medium conductivity $\sigma_{e}$ is an important parameter influencing the recruitment behavior.
\end{abstract}

\section{INTRODUCTION}

The control of complex motor tasks (such as hand grasp or walking) demands selective control of the individual muscles involved in the task. When using (artificial) electrical stimulation techniques, selectivity can be increased by positioning the stimulation electrodes close to the nerve fibers innervating the muscles to be controlled. Theoretically it would be possible to achieve maximal selectivity by using an intraneural stimulation prosthesis with as many electrodes as there are nerve fibers.

In a theoretical analysis Rutten et al. derived a cubic dependence of muscle force on stimulus current amplitude for monopolar stimulation in the low-force range: $F \propto I^{3}[1]$. They used a local approach and assumed a uniform distribution of nodes of Ranvier and an isotropic medium. Animal experiments generally confirmed this cubic dependence [1]; however, for some electrode positions the power of the stimulus current to which the force is proportional was found to deviate considerably from 3 . In experiments carried out by Meier the power was generally higher than 3 [2].

The main cause of this discrepancy might be the small number of nerve fibers innervating a muscle (e.g. \pm 69 for the extensor digitorum longus muscle (EDL) of the rat) in relation to the number of electrodes [1], [2], [3]. Therefore, the position of the stimulation electrode inside the fascicle (relative to the discrete positions of the nodes of Ranvier) is an important parameter determining the recruitment behavior in the low-force range. In addition, the distribution of the nerve fibers in the cross-sectional area of the fascicle is of importance: randomly in the whole area or clustered in a subsection of the area [4].

\section{EXPERIMENTS}

Acute experiments were performed on anaesthetized Wistar rats. A $25 \mu \mathrm{m}$-diameter wire electrode was cut at an angle of $45^{\circ}$ and inserted in the intact peroneal nerve of the right hindleg. Monophasic rectangular current pulses of 100 $\mu s$ duration were generated in order to stimulate $\alpha$-motoneurons of the EDL. The distal tendon was cut and connected to a force transducer. The elicited twitch contraction forces were measured under isometric conditions. For each electrode position two recruitment curves were recorded: one with the nerve suspended in air and one with the nerve immersed in Ringer.

The recruitment curves were plotted on a doublelogarithmic scale and parametrized by the threshold current and the slope in the low-force range (between 0.5 and 15 grams). The slope was determined by applying a linear fitting procedure to the double-logarithmic data. The threshold current was defined as the lowest stimulation current that resulted in a force level exceeding the lower fitting limit.

Fig. 1 shows a plot of threshoid current versus slope. Each data point corresponds to one recruitment curve. The plot contains pooled data from 6 rats for two external conditions: a nerve suspended in air and a nerve immersed in Ringer

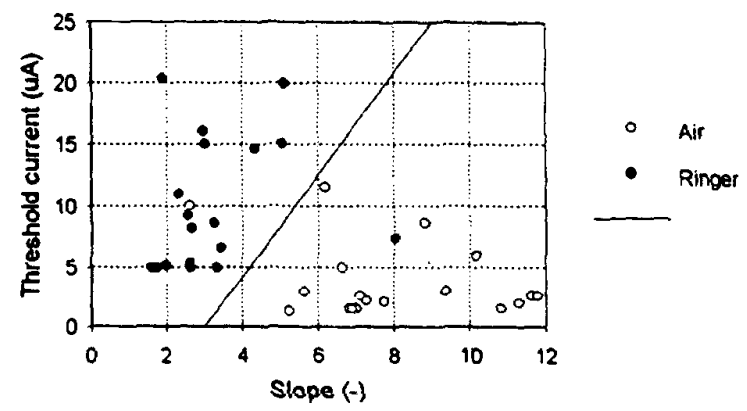

Fig. 1. Threshold current versus slope for 36 experimental recruitment curves. 18 points are shown for both a nerve suspended in air and a nerve immersed in Ringer. Each data point corresponds to one recruitment curve. The solid line dividing the data points into two groups is shown for clarity. 
(corresponding to $\sigma_{\mathrm{e}} \approx 0.0 \Omega^{-1} \mathrm{~m}^{-1}$ and $\sigma_{\mathrm{e}} \approx 1.65 \Omega^{-1} \mathrm{~m}^{-1}$, respectively).

From Fig. 1 it is clear that for a nerve suspended in air threshold currents are small and tend to be independent of the slope. For a nerve immersed in Ringer threshold currents show a considerable spread and tend to increase with the slope.

\section{Simulations}

To simulate intraneural stimulation the fascicle was modeled as a cylindrical volume conductor as described by Meier [2]. First the potential distribution was calculated for a monopolar stimulation electrode positioned inside the cylinder. Then the nerve fibers were placed in the model and their responses to the potential distribution were determined. In order to mimic the recruitment behavior of the EDL of the rat 69 nerve fibers were used with a total force contribution of 90 grams [3].

Recruitment curves were simulated both for a random distribution of the nerve fibers in the whole cross-sectional area of the fascicle and for a clustered distribution in a $25 \%$ subsection of this area. For both cases 20 electrode positions were chosen randomily. For each position 4 values of the external medium conductivity were evaluated: $\sigma_{e}=0.0,0.01$, 0.1 , and $5.0 \Omega^{-1} \mathrm{~m}^{-1}$. This resulted in 80 recruitment curves for both cases.

The recruitment curves were parametrized by the threshold current and the slope in the low-force range. Fig. 2 shows plots of threshold current versus slope. Each data point corresponds to one recruitment curve.

From Fig. 2 it is clear that for an insulating external medium $\left(\sigma_{\mathrm{e}}=0.0\right.$ and $0.01 \Omega^{-1} \mathrm{~m}^{-1}$ ) threshold currents are small and tend to be independent of the slope. For a (good) conducting external medium $\left(\sigma_{e}=0.1\right.$ and $\left.5.0 \Omega^{-1} \mathrm{~m}^{-1}\right)$ threshold currents show a considerable spread and tend to increase with the slope.

\section{Discussion}

The data points in Fig. 2 can be easily divided into two groups, corresponding to an insulating and a (good) conducting external medium, respectively. This is indicated by the solid lines in the plots. In the experimental results (Fig. 1) two groups can also be distinguished, corresponding to an insulating (air) and a good conducting (Ringer) external medium, respectively.

From Fig. 1 and Fig. 2, it is clear that the experimental results compare better with the simulation results for clustering of the nerve fibers than with the results for no clustering. This is most evident for the case of a (good) conducting external medium (as reflected by the orientation of the separation line between the two groups).

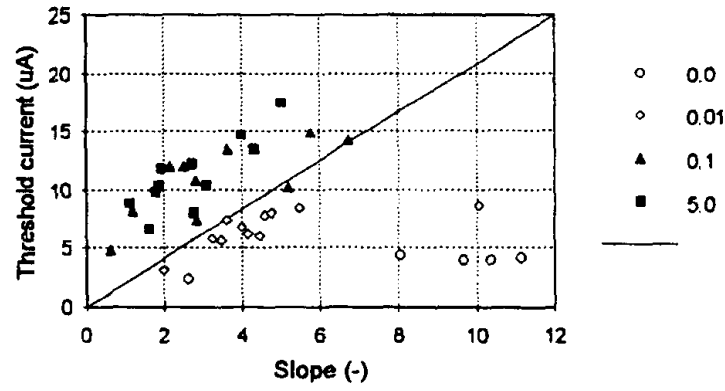

(a)

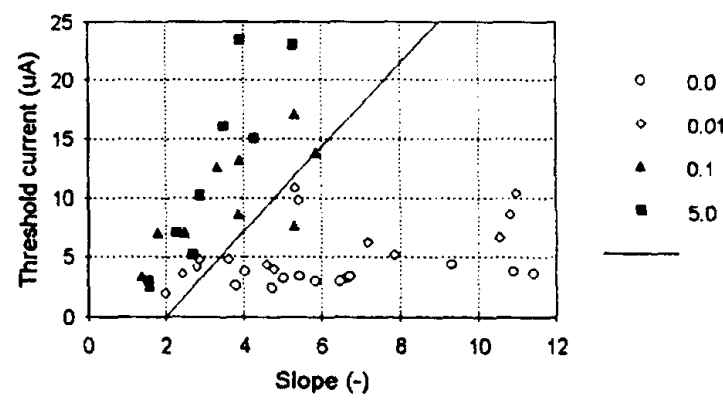

(b)

Fig. 2. Threshold current versus slope for simulated recruitment curves for a random distribution of the nerve fibers in the whole crose-sectional area of the fascicle (a) and for a clustered distribution in a $25 \%$-subsection of this area (b).

Points are shown for 4 values of the external medium conductivity $\sigma_{e}$. Each data point corresponds to one recruitment curve. The solid lines dividing the data points into two groups are shown for clarity.

\section{Conclusions}

From the results presented here, it can be concluded that both the low density of nodes of Ranvier (due to the small number of $\alpha$-motoneurons innervating a muscle) and the degree of clustering of nerve fibers are of major importance for the recruitment behavior resulting from intraneural stimulation. Furthermore, the external medium conductivity is an important parameter influencing the recruitment behavior. This has implications for the selectivity that can be attained when (insulating) cuffs around the nerve are used.

\section{REFERENCES}

[1] W.L.C. Rutten, H.J. van Wier, and J.H.M. Put, "Sensitivity and selectivity of intraneural stimulation using a silicon electrode array." IEEE Trans. Biomed. Eng., vol. 38, pp. 192-198, Feb. 1991.

[2] J.H. Meier, Selectivity and design of neuro-electronic interfaces, Ph.D. thesis, University of Twente, Enschede, The Netherlands, 1992.

[3] J.M. Peyronnard, L.F. Charron, J. Lavoie, and J.P. Messier, "Motor, sympathetic and sensory innervation of rat skeletal muscles," Brain Res. vol. 373, pp. 288-302, 1986.

[4] J.P.A. Smit, W.L.C. Rutten, T.A. Frieswijk, T.H. Rozijn, and R. Busschers, "The effect of spatial clustering of motor fibers in a nerve fascicle on force recruitment during electrical stimulation," in Proc. 14th Ann. Int. Conf. IEEE Eng. Med. Biol. Soc., pp. 1389-1391, 1992. 